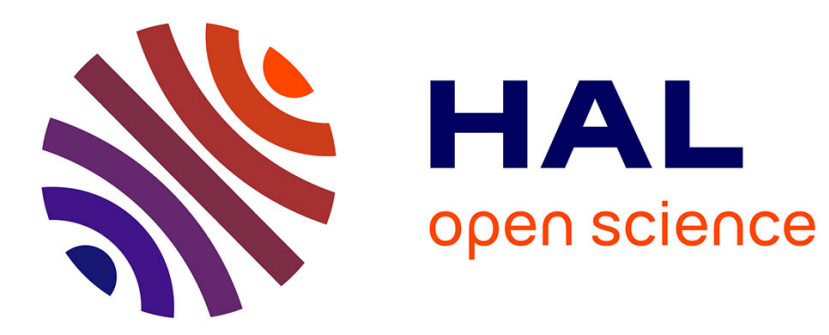

\title{
la culpabilité maternelle
}

Michèle Benhaim

\section{- To cite this version:}

Michèle Benhaim. la culpabilité maternelle. Revue de l'enfance et de l'adolescence RAFEF-GRAPE, 2002, 10.3917/lett.047.56 . hal-01429368

\section{HAL Id: hal-01429368 \\ https://hal-amu.archives-ouvertes.fr/hal-01429368}

Submitted on 7 Jan 2017

HAL is a multi-disciplinary open access archive for the deposit and dissemination of scientific research documents, whether they are published or not. The documents may come from teaching and research institutions in France or abroad, or from public or private research centers.
L'archive ouverte pluridisciplinaire HAL, est destinée au dépôt et à la diffusion de documents scientifiques de niveau recherche, publiés ou non, émanant des établissements d'enseignement et de recherche français ou étrangers, des laboratoires publics ou privés. 


\section{LA CULPABILITÉ MATERNELLE}

Michèle Benhaïm

\section{ERES | « La lettre de l'enfance et de l'adolescence »}

2002/1 n 47 | pages 49 à 56

ISSN 1146-061X

ISBN 2749200245

\section{Article disponible en ligne à l'adresse :}

http://www.cairn.info/revue-lettre-de-l-enfance-et-de-ladolescence-2002-1-page-49.htm

\section{Pour citer cet article :}

Michèle Benhaïm, "La culpabilité maternelle », La lettre de l'enfance et de l'adolescence 2002/1 ( $\left.\mathrm{n}^{\circ} 47\right)$, p. 49-56.

DOI 10.3917/lett.047.56

Distribution électronique Cairn.info pour ERES.

(C) ERES. Tous droits réservés pour tous pays.

La reproduction ou représentation de cet article, notamment par photocopie, n'est autorisée que dans les limites des conditions générales d'utilisation du site ou, le cas échéant, des conditions générales de la licence souscrite par votre établissement. Toute autre reproduction ou représentation, en tout ou partie, sous quelque forme et de quelque manière que ce soit, est interdite sauf accord préalable et écrit de l'éditeur, en dehors des cas prévus par la législation en vigueur en France. Il est précisé que son stockage dans une base de données est également interdit. 


\section{La culpabilité maternelle}

\section{Michèle Benhaïm}

Il y a une douzaine d'années, dans un ouvrage intitulé La folie des mères ${ }^{1}$, je tentais de mettre en lumière un fantasme maternel auquel serait confrontée chaque femme devenant mère et qui l'inscrirait dans la «folie ", non pas au sens d'une structure psychotique de la personnalité, mais comme passage inévitablement intriqué au processus de la maternité et qui s'énoncerait ainsi : le moment où une femme accède à la maternité dans le réel, c'est-à-dire ce temps de rupture dans le corps que signe l'accouchement, s'accompagnerait d'un temps de bascule imaginaire, fantasmatique. Devenir mère équivaudrait à devenir coupable d'avoir inscrit l'enfant dans l'ordre de la mortalité et donc, par conséquent, coupable de tout ce qui lui arriverait. Ce fantasme se déclinerait différemment et de façon variable selon son degré de proximité avec le conscient d'une part, selon le parcours historique de la mère dans son rapport à la place dévolue au père, d'autre part, ou, pour le dire encore autrement, dans son propre rapport à la question du manque.

À l'origine des positions subjectives maternelles, un « meurtre » imaginaire d'enfant circule. Ce qui fera peut-être mourir Jocaste (dont la tragédie commence, rappelons-le, par un abandon d'enfant que l'on voue à la mort) et qui permettra à Freud ${ }^{2}$ de repérer « une angoisse (chez l'enfant) d'être assassiné par la mère... ».

\footnotetext{
Michèle Benhaïm, psychologue, psychanalyste, chargée de cours à l'université de Provence, Aix-Marseille. Auteur de La folie des mères, Imago, 1992, 1998 ; Les troubles de la relation à la mère, Privat, 1992 ; Sida, luttes à vif (avec J. Broda), La pensée sauvage, 1994 ; L'ambivalence de la mère, Érès, 2001 ; Le regard d'Elsa, L'Harmattan, 2001.

1. M. Benhaïm, La folie des mères, Imago, 1992, 1998.

2. S. Freud, "Sur la sexualité féminine », dans La vie sexuelle, Paris, PUF, 1931.
} 
Avant de développer les liens qu'entretiennent maternel et culpabilité, et de cerner les éléments qui engendrent cette dernière, que nous repérons dans ce qui divise l'amour maternel pour en faire un sentiment ambivalent et que nous nommons haine, faisons un petit détour par l'histoire de ce concept.

\section{Freud et le sentiment de culpabilité}

Dans Malaise dans la culture ${ }^{3}$, Freud fait du sentiment de culpabilité une variété topique de l'angoisse devant la perte d'amour : si l'on est coupable de l'amour perdu, autrement dit de ne pas être aimé, alors la conscience de culpabilité se manifeste comme besoin de punition.

Ainsi, d'une part, la culpabilité s'originerait dans la renonciation à la satisfaction immédiate de la pulsion, d'autre part, le souhait interdit demeurant néanmoins, elle prendrait source dans le besoin de punition.

Ainsi, si l'on renonce aux satisfactions pulsionnelles, c'est pour ne pas perdre l'amour de l'objet. Et si le souhait persiste, survient le sentiment de culpabilité, comme si l'acte et l'intention étaient équivalents.

Toujours dans Malaise, Freud poursuit et trouve dans le complexe d'Edipe le fondement du sentiment de culpabilité relatif, en l'occurrence, au meurtre du père qu'accomplissent les frères. Pourtant, le mythe fondateur souligne bien comment ce sentiment de culpabilité précédait le meurtre et engendre, une fois l'acte accompli, le remords.

Si l'acte accompli repose sur la haine, le remords de l'acte témoigne de l'amour. Ainsi, sur cette ambivalence repose l'interdit posé sur la répétition de cet acte. Mais, et nous l'entendons évidemment dans notre clinique, si l'acte est interdit, l'intention demeure et avec elle persiste le sentiment de culpabilité.

Nous retiendrons que, pour Freud, le sentiment de culpabilité exprime un conflit d'ambivalence, reflet d'un éternel combat entre pulsion de vie et pulsion de mort.

Si, pour le cas de figure qui nous intéresse, le processus maternel, le sentiment de culpabilité relatif au remords de l'acte se laissent percevoir consciemment (par exemple dans les cas d'infanticide), le sentiment de culpabilité relatif à l'intention demeure inconscient et représente l'un des objets d'analyse avec les mères qui viennent nous dire une certaine souffrance à être mère, dont elles ne saisissent ni l'objet ni le sens.

Si l'on articule la logique freudienne autour de l'origine du sentiment de culpabilité avec la culpabilité maternelle qui, je le suppose, prend les traits d'une folie, on s'aperçoit en effet que, l'acte accompli ou pas, la mère se (re)trouve coupable. C'est un des éléments qui me permet de poser la question maternelle sous l'angle de l'ambivalence (que nous examinerons plus bas). C'est également un des aspects que souligne l'argument de ce numéro lorsqu'il évoque les professionnels de l'enfance confrontés aux parents « coupables » de ce qui arrive à leur enfant (maladie, etc.) et dont la lecture renvoie bien au fantasme de toute-puissance. En effet, que ce soit " grâce à moi » ou "à cause de moi », c'est bien au narcissisme primaire

3. S. Freud, Malaise dans la culture, PUF, $4^{\mathrm{e}}$ édition, 2000. 
que renvoie la position d'énonciation, et, secondairement, à un rapport particulier à la castration.

À cet endroit, les mères viennent nous adresser une plainte, celle d'échouer à être (toute) bonne. Ce moment correspond au temps d'une culpabilité auparavant latente, qui affleure le conscient et ainsi menace le sujet dans son intégrité moïque.

Reste alors à engager un travail d'analyse qui reposera sur la mise en lumière du fantasme sous-jacent de " meurtre », d' " infanticide " plus exactement, et de désintrication de ce qui relève d'une angoisse primordiale destructrice et d'une faute imaginaire.

Avant d'articuler ces différentes remarques à notre hypothèse sur l'ambivalence de la mère, voyons à présent le traitement que Winnicott fait de ce concept de culpabilité, l'un des points essentiels dans sa conceptualisation du développement de l'enfant.

\section{Winnicott et la capacité de sollicitude}

En l'opposant à la « sollicitude » qu'il envisage positivement, Winnicott ${ }^{4}$ fait de la culpabilité un phénomène négatif. Dans la (stricte) continuité de Freud, il fait dériver le sentiment de culpabilité de l'angoisse reposant sur l'ambivalence qui requiert à la fois le maintien de l'objet et l'idée de sa destruction. En quelque sorte, la sollicitude est la façon dont le sujet se sent « concerné », "impliqué », autrement dit « responsable », et en cela elle est bien positive ; elle prendrait l'aspect négatif au moment où ce sentiment de responsabilité basculerait dans un sentiment de culpabilité.

Chronologiquement, si Freud fait s'originer le sentiment de culpabilité dans le complexe d'Edipe, Winnicott le place au centre d'une relation duelle d'avant l'œdipe, c'est-à-dire dans la relation mère-enfant. Stade qui se caractérise en partie par l'ambivalence de l'enfant à l'égard de l'objet qu'il aime en même temps qu'il lui oppose des pulsions agressives et de destruction. Pour Winnicott, et mon hypothèse le rejoint sur ce point, l'ambivalence est une réussite à atteindre, puisqu'à partir d'elle s'élaborera « la capacité de sollicitude ». Au cours de cette expérience, l'enfant réunit et pacifie psychiquement deux imagos maternelles, "la mèreobjet » qui est susceptible de satisfaire les pulsions, on pourrait dire encore la mère réelle, celle de la toute-puissance, dont l'enfant autiste par exemple ne saurait se départir, et « la mère-environnement », celle qui contient, sécurise, soigne activement, bref la " mère suffisamment bonne ", la " juste assez mais pas trop ».

Que ces deux relations à la mère fusionnent résout l'ambivalence et permet à l'enfant un balancement tenable entre son angoisse de " dévorer » la mère puisqu'il l'aime, donc de la perdre, et sa capacité à la réparer, capacité susceptible de contenir cette angoisse. C'est cette angoisse contenue, c'est-à-dire qualitativement transformée, qui devient sentiment de culpabilité.

En cela la mère trouve à se nommer « environnement » puisque, pour la réparer, encore faut-il qu'elle assure une présence régulière. Ce sentiment de culpabilité tendra d'autant plus vers la sollicitude, c'est-à-dire vers la responsabilité, que

4. D.W. Winnicott, Processus de maturation chez l'enfant, 1962. 
les occasions de voir la mère non détruite par les pulsions de l'enfant se présenteront. À défaut, si l'objet ne survit pas à l'agression, c'est " l'effondrement » qui conduit l'enfant à y réagir en régressant, en l'occurrence à la phase d'omnipotence. Celle-ci prend alors les traits suivants : je suis coupable de la catastrophe dont je suis l'objet. Le traitement névrotique adulte qui en suivra verra des sujets en proie à un sentiment de culpabilité permanent dans lequel, pour lui donner sens, ils pourront inscrire de la haine.

\section{Culpabilité et maternité}

De la même façon que l'enfant attribue au même objet le «bon » et le « mauvais ", la mère dirigera sur le même objet, l'enfant, l'amour et la haine. Ici, l'enfant est appréhendé dans le lieu même où le place le discours de la mère, c'est-à-dire non pas comme objet réel, mais comme objet fantasmatique.

La culpabilité maternelle pourrait être ce sentiment qui entrave, en même temps qu'il l'engendre, la reconnaissance d'une ambivalence nécessaire à l'élaboration de la séparation, coupure symbolique à l'origine de la subjectivité de l'enfant et du (ré)investissement du champ du féminin pour la mère.

Ce que révèle la clinique des mères, autant au travers des psychothérapies, des analyses qu'au cours d'épreuves projectives ${ }^{5}$, et plus exactement ce qu'illustre le registre identificatoire convoqué à cette occasion, c'est que dans le destin maternel, la « responsabilité » tend à se confondre avec la «culpabilité ». Au-delà de la superposition partielle et/ou temporaire à l'œuvre dans le parcours maternel commun et quotidien, la psychopathologie peut témoigner d'une superposition pouvant atteindre la totale confusion, comme essentiellement dans les cas d'infanticides.

En effet, en donnant la mort à l'enfant, la mère révèle parfois le sentiment de se supprimer, sorte de suicide altruiste ou de meurtre de la mère en elle, et qui correspond à ce temps fugace dans lequel s'inscrit en général l'acte meurtrier, survenant souvent dans une confusion absolue entre l'autre (l'enfant) et soi.

Les discours, les lapsus, les craintes plus ou moins conscientes, les rêves, les cauchemars des mères viennent confirmer, témoigner de ce qui, ailleurs, dans le champ de la psychopathologie, apparaît de façon plus manifeste : l'expression d'une faute imaginaire, l'expression d'une faute ayant été commise par quelqu'un d'autre, le sentiment de culpabilité qui lui est étroitement associé et le « besoin de punition » qui s'avère relever ici d'un appel, d'une demande thérapeutique.

Certains parcours trouvant une issue dans l'IVG, certains deuils inélaborables d'enfants morts dans le réel, l'infanticide enfin, en sont les supports privilégiés.

La clinique du discours maternel reflète parfois un sentiment de culpabilité qui recouvre la quasi-certitude d'être coupable d'un meurtre dans la réalité. Ainsi de Sandrine qui perd un enfant dans un accident où elle n'était pas présente et dit : «Ayant provoqué la mort d'un enfant, je n'avais plus le droit d'en avoir d'autres. » Plus loin elle précise : «Je l'ai tué dans le sens où je ne l'ai pas secouru. » Ne pas sauver l'enfant, c'est le tuer. Ne pas poser un acte devient un acte en soi pour jus-

5. M. Benhaïm, « L'ambivalence maternelle à l'épreuve du Patte-Noire », dans L'infantile, n 1, octobre 2001. 
tifier la culpabilité et lui donner de la sorte un objet. Là se révèle la confusion entre ce qui pourrait relever d'une faute objectivable, en réalité inexistante, et ce sentiment de culpabilité intériorisé bien auparavant, latent et révélé ici brutalement à la conscience, en tout cas étrange et complexe.

Ainsi de Chloé, mère d'une fillette handicapée et dont les propos indiquent que, plutôt que de garantir son enfant comme sujet, elle s'attache à constater un dommage causé à ce dernier (à elle-même ?) et tente alors d'établir un lien entre ce dommage et une faute qu'elle n'aurait pas commise, nous demandant à travers cette dénégation de dénouer ce qui, au-delà de la culpabilité, au travers de l'ambivalence, peut se vivre malgré tout d'un amour maternel pas trop surchargé de " remords".

L'infanticide pourrait, sous cet angle, recouvrir la faute (commise cette fois) afin que le lien entre celle-ci et le « dommage » devienne une certitude et entérine sans faille la culpabilité maternelle : "Je suis bien toute-puissante, je ne connais pas le manque ", culpabilité qui, à ce prix, permettrait l'économie psychique de la reconnaissance de l'ambivalence du sentiment maternel.

Que la mère évolue du côté de l'amour-tout ou du côté de la haine-toute, on voit bien comment l'un comme l'autre sont mortifères dans leur totalité : ce qui se joue sans doute, c'est l'impossible d'une séparation annoncée par Cathy lorsqu'elle évoque « la perte de l'odeur de bébé » comme déclenchant la pulsion meurtrière, que signe une haine non symbolisable, « je lui consacrais tout mon temps ». La fusion dérive à cet endroit : de " responsable » d'un autre, Cathy devient coupable de sa mort comme elle l'était probablement de sa vie.

La clinique montre comme cela, au quotidien, comment le sentiment de culpabilité, que cause en même temps qu'il cause la haine, se noue toujours pour chacune, de façon singulière, mais vraisemblablement incontournable.

Francine l'exprime à travers un état quasi dépressif : " J'ai peur de le perdre, je me sens impuissante s'il lui arrive quelque chose.»

Le fantasme " un enfant est en danger de mort » alimente ainsi couramment les propos maternels, fantasme intriqué avec celui d'une mère impuissante à le sauver. De quel enfant s'agit-il ? Celui qu'elle a été ? Que ou qui n'a-t-elle pas secouru ? "Sans moi, il serait seul et perdu ! Il faudrait qu'il apprenne à se débrouiller seul. »

Alternance des registres psychiques pouvant contenir la culpabilité soit sur le versant de la faute (ce n'est pas moi, c'est l'autre), soit reposant sur ce qui relève du propre rapport maternel au manque : c'est l'objet qui est perdu à jamais, l'enfant, lui, à ce prix, peut y retrouver sa route.

La clinique de la culpabilité maternelle met à jour l'ambivalence amour/haine. L'hypothèse se précise : la culpabilité, le sentiment de culpabilité, s'il permet de reconnaître l'ambivalence, c'est-à-dire que de la haine traverse l'amour maternel et ainsi en tempère les excès, pourrait recouvrir le concept de sollicitude winnicottien, qui verrait là une mère ayant quitté sans remords le stade de préoccupation maternelle primaire et devenir responsable d'un autre, radicalement autre dont elle peut donc s'occuper dans l'alternance de sa présence-absence et qu'il lui survive, autrement dit dont elle peut se séparer.

En revanche, si d'avoir été prise auparavant dans une élaboration impossible de sa castration, d'avoir donc été figée dans une position narcissique ne pouvant 
entrevoir dans le processus qui conduit à être mère qu'une unité mère-enfant, finalement si rien n'autorise ce processus de reconnaissance et d'acceptation de son ambivalence, les demandes émergent alors de mères ployant de façon dépressive sous le poids d'une faute imaginaire indicible, dont elles sont coupables toujours et pour toujours.

Si alors, fort heureusement, pas toutes ne vont jusqu'à lui donner un objet (infanticide), des symptômes autres peuvent en devenir l'expression et nous avons pu en repérer des éléments dans, notamment, le quotidien de mères d'adolescents, les contextes de maltraitances, voire dans d'impossibles conceptions d'enfants...

\section{Ambivalence maternelle}

Chez Freud ${ }^{6}$, nous pouvons repérer l'origine de l'ambivalence définie comme l'observation possible d'une motion pulsionnelle à côté de son contraire, dans un des destins possibles des pulsions : le renversement de contenu, dont la seule occurrence, précise encore Freud, est la transformation de l'amour en haine.

" Tout se passe comme si la complémentarité même du souhait incestueux et du souhait de mort suscitait un troisième souhait destiné à garantir leur innocuité, souhait opposé au souhait de mort et qui se présente sous la forme de l'attente d'un châtiment ou d'une séduction amoureuse de la part de celui dont on souhaite la mort ", nous dit Conrad Stein 7.

C'est dans son texte Deuil et mélancolie ${ }^{8}$ que Freud fait apparaitre l'occasion privilégiée de révéler cette ambivalence contenue dans les relations d'amour, en décrivant la situation de perte de l'objet d'amour où l'on est « soi-même responsable de la perte de l'objet d'amour, autrement dit, on l'a voulue ». Dans son étude sur les Névroses de guerre ${ }^{9}$, il avait étudié les effets de l'agression externe et du danger de mort sur des sujets particulièrement exposés. Cette étude l'a conduit à s'interroger sur l'énigme de la répétition, le sujet répétant une situation désagréable pour en avoir la maîtrise. Ainsi de son observation de ce que l'on nomme depuis le « fort-da " pour montrer comment la dramatisation scénique introduit une « liaison de la pulsion de mort à la pulsion de vie ». Au cours du jeu, en effet, l'enfant effectue un travail qui rend possible l'élaboration psychique de la perte d'objet. À la suite d'une perte de soins maternels, d'une perte de sa présence, Ernst tente une maîtrise symbolique de l'objet perdu et, pour ce faire, d'objet aimé et passif de soins, il se fait actif, c'est-à-dire sujet de l'appel, de la demande, se constituant ainsi comme sujet aimant : dans ce renversement dialectique qui le sauve, Ernst donne corps à l'absence de l'objet en l'évoquant. Il se nomme objet perdu pour la mère.

L'hypothèse autour de l'ambivalence de la mère s'inscrit au détour du jeu du « fort-da » dans une proposition qui pourrait s'énoncer ainsi : puisque la mère ne peut « perdre » l'enfant qu'en s'absentant vraiment, l'enfant tente de sauver son

\footnotetext{
6. S. Freud (1915), « Pulsions et destins des pulsions », dans Métapsychologie, Gallimard, 1968.

7. C. Stein, L'enfant imaginaire, Denoël, 1971.

8. S. Freud (1915), « Deuil et mélancolie », dans Métapsychologie, Gallimard, 1968.

9. S. Freud (1918), «Introduction à la psychanalyse des névroses de guerre », dans Résultats, idées, problèmes, I, PUF, 1984.
} 
désir en indiquant à la mère que c'est dans sa présence même qu'il lui faut le quitter, c'est-à-dire s'absenter, certes, mais à lui, et dans une continuité de sa présence. Autrement dit, l'élaboration de la haine qui autoriserait l'enfant à emprunter les voies de la subjectivation relèverait de la séparation, de la coupure symbolique et non de la coupure réelle, du clivage radical.

Freud soulignait bien le rôle de continuité d'être que l'environnement joue pour l'enfant. Enfant pouvant être pris dans l'angoisse, soit dans une situation de danger où cette angoisse relèverait d'une crainte de voir survenir une expérience d'impuissance, soit dans une situation traumatique où elle serait liée à un abandon qui verrait le sujet en être submergé. Et si « la mort que l'on tue par le silence provoque un appauvrissement de la vie 10 », le rapport au «vrai » pourrait à l'inverse se dire dans une présence maternelle sur fond d'absence et dans une absence de la mère sur fond de permanence et de continuité.

Pour Freud, l'acte primordial n'est pas, comme on pourrait le penser au premier abord, le meurtre du père, mais bien la mise au monde hors du ventre de la mère. D'ailleurs, " l'attribution de la procréation au père ne peut être que l'effet d'un pur signifiant », souligne Lacan. Et Freud, ajoute-t-il, a été amené à « lier l'apparition du signifiant du père, en tant qu'auteur de la loi, à la mort, voué au meurtre du père, montrant ainsi que si ce meurtre est le moment fécond de la dette par où le sujet se lie à vie à la loi, le père symbolique en tant qu'il signifie cette loi est bien le père mort ${ }^{11}$ ».

On peut y lire une représentation de la toute-puissance et de l'éternité : l'enfant ne tient son existence que de la sortie du ventre de sa mère.

La séparation, produit de l'élaboration, maternelle et par conséquent de l'enfant, de la haine, relève d'un don de vie au sens d'une transmission de vie.

Si l'on occulte cette dimension de transmission, on risque de confondre don et dette, et nous savons combien la reconnaissance de la dette s'accomplit le plus souvent dans une culpabilité névrotique.

De même que le concept de culpabilité inconsciente se réfère à la limitation de la toute-puissance du désir qui anime les vœux, " pour autant que cette limitation se révèle sous la forme de la visée d'être reconnu coupable », de même l'idée d'une ambivalence de sentiments inconscients relève du fait que la toute-puissance du désir est limitée justement parce que le désir investit simultanément deux vœux dont les contenus sont contradictoires. D'ailleurs, les concepts de culpabilité inconsciente et d'ambivalence des sentiments, on l'a vu, se recouvrent dans la mesure où le souhait d'être " condamné », puni et séduit par celui-là même dont on souhaite la mort, ce souhait contrecarre le vœu de mort en question.

Aimer seulement comme haïr seulement n'inscrivent pas l'enfant dans le désir de l'Autre, mais le soumettent au «caprice » maternel et relèvent, l'un comme l'autre, d'une économie psychique à laquelle la mère sacrifie l'enfant et qui lui évite de se confronter à la perte en étant maternelle dans un cas comme dans l'autre, sur le mode de la toute-puissance.

Amour, haine, ambivalence, sous un angle maternel inconscient, ce que l'on veut, sous couvert du souhait conscient " éviter que l'enfant ait mal ", c'est 
contourner le sentiment de culpabilité dans lequel la régression maternelle, nécessaire à la survie du bébé, nous entraîne inévitablement.

\section{Conclusion}

La haine constitue un point d'impasse si elle est envisagée dans sa seule dimension imaginaire, c'est-à-dire dans ses effets destructeurs, non dans sa dimension symbolique où, au contraire, elle inscrit l'impossible de la fusion mèreenfant et permet ainsi à l'agressivité de ne pas basculer dans le registre de la violence. La maternité convoque la question de la jouissance dans ce moment de traversée du réel qu'est l'enfantement proprement dit. Elle peut n'être qu'un passage, et c'est bien sûr le cas en général, le narcissisme à l'œuvre ne masquant qu'illusoirement la castration vite rappelée au bon souvenir de la mère par l'altérité immédiate et radicale de l'enfant ; elle peut, et ce sont ces discours maternels coupables que la clinique nous donne à entendre, faire que la mère demeure captive d'une fascination : celle de l' « enfant-mort ». Là, toute situation de séparation, et l'accouchement l'inaugure, ramène cette mère à « cette catastrophe identitaire première » où s'origine le passage à l'acte dans le réel.

Si le réel de la haine, c'est l'impossible de la fusion, le réel de la fusion, cet évitement radical de la culpabilité et de l'ambivalence, défaut qui prive le sujet d'effets structurants au risque de son anéantissement, le réel de la fusion donc, c'est la mort. Jean-Paul illustre et conclut notre propos, lui qui, matricide, explique : «De nous trois, mon frère, ma sœur et moi, j'étais le plus proche de ma mère, donc seul moi pouvais accomplir ce geste... » 